\title{
AVALIAÇÃO DO USO DE MEDICAMENTOS PELA POPULAÇÃO IDOSA EM MONTES CLAROS, MINAS GERAIS, BRASIL
}

\author{
Evaluation of medication use among elderly population \\ Evaluación del uso de los medicamentos por la población anciana
}

\author{
Carla Silvana Oliveira Silva ${ }^{1}$ \\ Maria Ivanilde Pereira ${ }^{2}$ \\ João Felício Rodrigues Neto ${ }^{4}$ \\ Dulce Aparecida Barbosa ${ }^{5}$
}

Aparecida Yoshie Yoshitome ${ }^{3}$

\section{RESUMO}

Inserido no universo do cuidado de enfermagem à pessoa idosa, o estudo descreve o perfil sociodemográfico e verifica o uso de medicação segundo gênero em idosos cadastrados em um PSF. Método: Estudo quantitativo, descritivo, transversal com pesquisa de campo. Utilizou-se o questionário semiestruturado, possibilitando identificar o perfil desses usuários, as principais morbidades, os medicamentos utilizados e as dificuldades relatadas por eles quanto ao uso dos medicamentos. 0 universo foi de 211 idosos. Na análise dos dados utilizou-se o programa SPSS, priorizando a associação estatística de forma analítica. Resultados: Entre os idosos em uso de medicamentos, verificou-se predominância feminina, renda familiar e escolaridade baixas, idosos vivendo com familiares e sem dificuldades no uso de medicamentos. Uso de medicamentos foi comum em mais de $90 \%$ dos idosos. Verificou-se associação para "ausência de dificuldades no uso do medicamento" e "orientações do PSF" (P<0,001). Conclusão: Constatou-se que as mulheres encontram-se em situações menos privilegiadas.

Palavras-chave: Idoso. Uso de Medicamentos. Atenção Primária à Saúde. Enfermagem Geriátrica.

\begin{abstract}
Inserted in the care universe of nursing to the elderly people, the study describes the socio-demographic profile and verifies the medication use according to gender among elders from a Family Health Program unit. Method: This is a quantitative, descriptive and cross-sectional study with field research. It was used a semi-structured questionnaire, enabling to identify the profile of these users, main morbidities, used medicaments and related difficulties by them regarding the medication use. The research universe was 211 elders. In the date analysis was used SPSS software, prioritizing analytic statistic association. Results: Among elderly in medication use verified female predominance, low family income and low schooling and elders living with their familiars and without difficulties in medication use. The medication use was common in moreover $90 \%$ of elders. It was verified association to "lack of difficulties in medication use" and "Family Health Program orientations" ( $p<0,001)$. Conclusion: It was verified that the women are in low privileged situation.
\end{abstract}

Keywords: Aged. Drug Utilization. Primary Health Care. Geriatric Nursing.

\section{Resumen}

Inserido en el universo del cuidado de enfermería al anciano, el estudio describe el perfil sociodemográfico y verifica el uso de medicación según género en acianos asistidos en una unidad del Programa de Salud Familiar. Método: Estudio cuantitativo, descriptivo y transversal con trabajo de campo. Fue utilizado cuestionario seme-estructurado, posibilitando identificar el perfil de los usuarios, las principales morbilidades, los medicamentos utilizados y las dificultades relatadas por ellos en relación al uso de los medicamentos. En el análisis de los dados, fue utilizado el programa SPSS, priorizando la asociación estadística de forma analítica. Resultados: Entre los ancianos con uso de medicamentos, fue verificado predominancia femenina, renda familiar y escolaridad bajas, ancianos viviendo con los familiares y sin dificultades en uso de los medicamentos. El uso de medicamento fue común en más de $90 \%$ de los ancianos. Fue verificado asociación para "ausencia de dificultades en uso de medicamento" y "orientaciones del Programa de Salud Familiar" ( $p<0,001)$. Conclusión: Fue constatado que mujeres están en situación menos privilegiada.

Palabras clave: Anciano. Utilización de Medicamentos. Atención Primaria de Salud. Enfermería Geriátrica.

\footnotetext{
${ }^{1}$ Especialista em Enfermagem e Saúde Pública, docente do Departamento de Enfermagem da Universidade Estadual de Montes Claros - UNIMONTES. Montes Claros-MG. Brasil. E-mail: caralsosilva@ig.com.br, ${ }^{2}$ Mestre em Economia pela UFMG, com área de concentração em Economia da Saúde, docente do Departamento de Saúde Mental e Coletiva e do Departamento de Economia da UNIMONTES. Montes Claros-MG. Brasil. E-mail: ivanildps@yahoo.com.br , 3 Mestre em Enfermagem, enfermeira do Departamento de Enfermagem da Universidade Federal de São Paulo - Unifesp. São Paulo-SP. Brasil. E-mail: ayoshitome29@unifesp.br, ${ }^{4}$ Médico, Doutor em medicina. Coordenador do Programa de Pós-Graduação em Ciências da Saúde. Docente da Unimontes. Montes Claros-MG. Brasil. E-mail: joao.felício@unimontes.br , ${ }^{5}$ Pós-Doutora em Enfermagem, Docente da Universidade Federal de São Paulo. São Paulo - SP. Brasil. E-mail: dulce.barbosa@unifesp.br
} 


\section{INTRODUÇÃO}

A velhice é o último período da evolução natural da vida e caracteriza-se por um conjunto de parâmetros biológicos, econômicos, políticos e sociais que compõem 0 cotidiano das pessoas que vivem esta fase.

A Organização das Nações Unidas (ONU) estabeleceu, em 1985, a idade de 65 anos para definir o idoso nos países desenvolvidos. Entretanto, para os países em desenvolvimento onde a expectativa de vida é menor, adota-se 60 anos, como o Brasil, cujo Estatuto do Idoso referenda esta determinação ${ }^{1}$.

0 envelhecimento populacional decorre da diminuição das taxas de mortalidade e fecundidade, fenômeno que foi inicialmente observado em países desenvolvidos, com a urbanização adequada das cidades, melhoria das condições sanitárias ambientais, tanto residenciais como de trabalho e nutricional, além da elevação dos níveis de higiene pessoal ${ }^{2}$. Assim, o aumento da expectativa de vida e redução das taxas de mortalidade decorreram antes da introdução dos avanços na área da saúde ${ }^{3}$.

Nos países em desenvolvimento, a população idosa vem aumentando de forma rápida, mesmo sem ter resolvido questões econômico-sanitárias básicas, graças ao acesso às conquistas, como a descoberta das vacinas e antibióticos ${ }^{4}$.

No Brasil, o envelhecimento começa a ser observado, a partir de 1960, quando a queda da taxa de fecundidade começou a alterar sua estrutura etária, estreitando progressivamente a base da pirâmide populacional. A cada ano, 650 mil idosos são incorporados à população, passando de três milhões, em 1960, para sete milhões em 1975 e 17 milhões em 2006, o que representa um aumento de $600 \%$ em menos de 50 anos $^{5}$.

Esse quadro ocasiona inúmeros problemas, entre eles o aumento de gastos na área da saúde, uma vez que as pessoas nessa faixa etária estão mais susceptíveis a doenças e problemas psicossociais, assim como ao decréscimo das condições financeiras ${ }^{6}$.

Outro desafio advindo do envelhecimento populacional é o aumento dos problemas de saúde crônicos e degenerativos, relacionados aos sistemas cardiocirculatórios, neuropsiquiátricos, digestivos e osteoarticular, tornando os idosos passíveis de acometimento simultâneo de disfunções em diferentes órgãos.

0 surgimento de pluripatologias leva os idosos ao consumo de quantidade considerável de medicamentos, ${ }^{6}$ que passam a fazer parte de seu cotidiano. Gerenciar tais situações requer alternativas individualizadas. Cabe ressaltar que a utilização criteriosa e cautelosa dos medicamentos, seu uso correto - dose, tipo e intervalos - e a orientação adequada são alguns dos elementos essenciais para a prevenção da iatrogenia medicamentosa ${ }^{7}$.

Quase sempre há uma seleção variada de medidas não farmacológicas, dirigidas a um estilo de vida mais saudável. 0 emprego dessas medidas deve ser considerado, assim, como o acompanhamento com revisão periódica do conjunto dos medicamentos e de seus efeitos adversos, privilegiando o uso de monodrogas e fármacos de eficácia comprovada por meio de evidências científicas. Sempre que necessário, devese realizar a suspensão do uso medicamentoso, verificar a compreensão da prescrição e das orientações farmacológicas ou não farmacológicas e simplificar os esquemas de administração ${ }^{8}$.

Os idosos são os principais consumidores e maiores beneficiários da terapia medicamentosa moderna. Estima-se que $23 \%$ da população brasileira consumem $60 \%$ da produção nacional de medicamentos e que 64,5 milhões de pessoas em condições de pobreza não têm como custear suas necessidades básicas, tampouco têm acesso aos medicamentos, excetuandose os da rede pública, e que mais de $80 \%$ tomam, pelo menos, um medicamento diariamente 9 .

Os desdobramentos adversos da polifarmacoterapia favorecem sinergismos e antagonismos não desejados, descumprimento das prescrições dos produtos clinicamente essenciais e gastos excedentes com os de uso supérfluo ${ }^{8}$. Infelizmente, as substâncias farmacologicamente ativas nem sempre atingem seus objetivos com a eficácia desejada; por isso, busca-se cada vez mais utilizar novas estratégias de cuidado do idoso, de forma a garantir saúde e qualidade de vida para esse grupo etário.

A prescrição de medicamentos para essa população envolve necessariamente 0 entendimento das mudanças estruturais ou funcionais dos vários órgãos e sistemas relacionados com a idade, implicando alterações na farmacocinética e farmacodinâmica para vários medicamentos ${ }^{10}$. Esta tem sido uma prática médica comum nos serviços de saúde, sobretudo no serviço público. Nesse contexto, a enfermagem no trato com o idoso procura adotar o exercício da interdisciplinaridade, compondo as equipes de saúde da família e procurando dinamizar a atenção ao idoso.

Nesta prescrição, deve-se considerar, além das peculiaridades da farmacocinética e farmacodinâmica dos medicamentos, o custo da manutenção terapêutica e as dificuldades em se obter adesão ao tratamento, quase sempre agravado pelo "déficit" de memória, visual e presença de osteoartrite, dificultando a distinção e a manipulação de diversos medicamentos simultaneamente. A utilização correta de medicação 
por idosos pode aumentar a incidência de efeitos colaterais e interações medicamentosas, e o uso inadequado, com frequência, provoca complicações graves. ${ }^{11} 0$ uso inadequado de medicamentos por idosos vem se tornando um problema, tanto do ponto de vista humanístico como econômico, uma vez que existem evidências de que a população idosa gasta percentuais significativos de sua renda com medicação, o que, além de comprometer financeiramente o idoso, pode gerar outros problemas de saúde em função do emprego indiscriminado de medicamento.

Em contrapartida ao benefício da farmacoterapia, os idosos apresentam os maiores riscos de desenvolver reações adversas aos medicamentos, e estas são responsáveis por $10 \%$ a $20 \%$ das admissões hospitalares agudas. Muitas vezes, ao usar o medicamento, o idoso ou a família que o administra não segue a prescrição, a distribuição de horário e dosagens, além de ter crenças populares quanto a determinados tipos e formas de uso de fármacos (como restrições de misturas, de ingestão de alimentos associados a alguns alimentos, crenças que acabam interferindo nos efeitos esperados, aumentando, muitas vezes, a possibilidade de reações colaterais, prejudicando seu uso correto) $)^{10}$.

Diante da oportunidade de assistir os idosos em uso de medicamentos, pode-se observar que, na maioria das vezes, estes apresentam dificuldades no uso da medicação. A observação desses fatos ensejou a seguinte indagação: os idosos cadastrados em um PSF da cidade de Montes Claros-MG utilizam de forma correta a medicação prescrita?

Assim sendo, um estudo do uso dos medicamentos por pessoas idosas é relevante e necessário, pois a velhice traz consigo o desenvolvimento de multipatologia, a qual se torna crônica e exige um contínuo acompanhamento da equipe de saúde para proporcionar um tratamento eficaz na prevenção de complicações patológicas. 0 número de idosos em uso de medicamentos vem aumentando, tornando-se parte do grupo etário mais medicalizado da sociedade, sobretudo em razão do aumento da prevalência de doenças crônicas para a idade ${ }^{11}$. É importante também realizar estudos de avaliação desse uso a fim de elucidar algumas questões pertinentes e apresentar subsídios para a melhor compreensão dessa problemática. Este estudo tem como objetivos descrever o perfil sociodemográfico e verificar o uso de medicação, segundo gênero, na população idosa cadastrada em um Programa Saúde da Família (PSF), na cidade de Montes Claros-MG.

Espera-se que este estudo possa contribuir para um melhor conhecimento sobre o trato do idoso com o medicamento e, ao mesmo tempo, oferecer aos serviços de saúde novas estratégias para melhorar a atenção prestada à população idosa, sobretudo no que se refere às práticas medicamentosas, acompanhadas de medidas relacionadas às práticas saudáveis de vida.
Silva CSO, Pereira MI, Yoshitome AY, Neto JFR, Barbosa DA

\section{MÉTODO}

Trata-se de um estudo transversal, de abordagem quantitativa, descritiva, realizada em uma Unidade do Programa de Saúde da Família (PSF) na cidade de Montes Claros-MG.

0 universo contemplou 228 idosos registrados no Sistema de Informação de Atenção Básica (SIAB), identificados nas Fichas de Cadastro das Famílias - Ficha A. Participaram do estudo 211 idosos que atenderam os seguintes critérios de inclusão: aceitar participar da pesquisa; estar cadastrado no SIAB durante o período de coleta de dados; ser encontrado na residência em até duas tentativas; continuar residindo na área de abrangência da unidade de saúde pesquisada. Não houve perda de participantes durante o estudo.

0 instrumento utilizado para a coleta de dados foi o questionário semiestruturado aplicado aos idosos no período de julho a dezembro de 2008. Realizou-se um pré-teste com seis usuários idosos (três atendidos pelo PSF e três, pelas UBS da Cidade de Montes Claros) com o objetivo de observar possíveis falhas no instrumento e poder realizar as adequações necessárias. 0 questionário contemplou variáveis sociodemográficas e relativas ao uso de medicamentos, tais como: orientação sobre medicação, dificuldades em seu uso, conhecimento sobre o medicamento em uso e classificação das medicações quanto ao caráter contínuo ou usual.

Antes de ser realizada a coleta de dados no domicilio dos idosos, o projeto de pesquisa foi submetido ao Comitê de Ética e Pesquisa (CEP) da Universidade Estadual de Montes Claros e aprovado pelo Parecer Consubstanciado n. ${ }^{\circ}$ 655/07 e pelo CEP da UNIFESP por meio do Parecer $n .{ }^{\circ}$ 1366/08. Os idosos que concordaram participar do estudo assinaram o Termo de Consentimento Livre e Esclarecido antes de responderem ao questionário, visando a atender aos aspectos éticos e legais, estipulados pela Resolução $n{ }^{\circ}$ 196 de 1996 do Conselho Nacional de Saúde.

Os dados obtidos foram analisados utilizando-se o software SPSS Versão 10.6 e a análise estatística descritiva. 0 perfil dos idosos da amostra estudada foi descrito de acordo com as variáveis quantitativas que foram descritas por meio da média e desvio-padrão, e para as qualitativas foram utilizadas as frequências absolutas e relativas (percentagens). A quantidade de medicamentos utilizada pelos idosos e sua associação com características sociodemográficas foram analisadas por meio das técnicas de inferências: Teste de homogeneidade do Qui-quadrado, Análise de Variância (ANOVA) e teste de Mann-Whitney. 0 uso de medicamentos, a orientação recebida e as dificuldades no uso foram analisadas por meio do Odds ratio e o intervalo de confiança segundo o gênero dos idosos. Adotou-se o intervalo de confiança de $95 \%$. 
Avaliação do uso de medicamentos pelo idoso

\section{RESULTADOS}

Tabela 1 - Distribuição dos Idosos Cadastrados em PSF na cidade de Montes Claros segundo perfil demográfico e socioeconômico - Montes Claros, 2008

\begin{tabular}{|c|c|c|c|c|c|}
\hline \multirow[t]{3}{*}{ Variável } & \multicolumn{4}{|c|}{ Gênero } & \multirow{3}{*}{ p-valor } \\
\hline & \multicolumn{2}{|c|}{ Masculino } & \multicolumn{2}{|r|}{ Feminino } & \\
\hline & $\mathbf{n}$ & $\%$ & n & $\%$ & \\
\hline \multicolumn{6}{|l|}{ Fa ixa etária } \\
\hline 60 a 69 anos & 47 & $51,1 \%$ & $\begin{array}{l}6 \\
6\end{array}$ & $55,0 \%$ & \multirow{3}{*}{0,851} \\
\hline 70 a 79 anos & 34 & $37,0 \%$ & $\begin{array}{l}4 \\
1\end{array}$ & $34,2 \%$ & \\
\hline Acima de 79 anos & 11 & $12,0 \%$ & $\begin{array}{l}1 \\
3\end{array}$ & $10,8 \%$ & \\
\hline \multicolumn{6}{|l|}{ Estado civil } \\
\hline Casado & 57 & $62,0 \%(4,4)$ & 38 & $31,7 \%$ & \multirow{3}{*}{$<0,001$} \\
\hline Solteiro/separado/divorciado & 8 & $8,7 \%$ & 22 & $18,3 \%(2,0)$ & \\
\hline Viúvo & 27 & $29,3 \%$ & 60 & $50,0 \%(3,0)$ & \\
\hline \multicolumn{6}{|l|}{ Escolaridade } \\
\hline Alfabetiza do & 54 & $58,7 \%$ & 46 & $38,3 \%$ & \multirow{3}{*}{0,003} \\
\hline Analfabeto & 38 & $41,3 \%$ & 74 & $61,7 \%$ & \\
\hline \multicolumn{5}{|l|}{ Renda familiar } & \\
\hline Mais de 2 S.m & 14 & $15,2 \%$ & 14 & $11,7 \%$ & \multirow{3}{*}{0,449} \\
\hline Até 2 S.m & 78 & $84,8 \%$ & 106 & $88,3 \%$ & \\
\hline Não & 59 & $75,6 \%$ & 81 & $75,0 \%$ & \\
\hline \multicolumn{6}{|l|}{ Com que m o Sr(a). mora? } \\
\hline Sozinho & 16 & $19,5 \%$ & 23 & $19,7 \%$ & \multirow{8}{*}{0,001} \\
\hline Esposa & 33 & $40,2 \%(3,2)$ & 23 & $19,7 \%$ & \\
\hline Filho & 8 & $9,8 \%$ & 35 & $29,9 \%(3,4)$ & \\
\hline Irmão & 2 & $2,4 \%$ & 3 & $2,6 \%$ & \\
\hline Neto & & & 7 & $6,0 \%$ & \\
\hline Esposa e filho & 7 & $8,5 \%$ & 3 & $2,6 \%$ & \\
\hline Filho e neto & 4 & $4,9 \%$ & 8 & $6,8 \%$ & \\
\hline Outro/ outras situações & 12 & $14,6 \%$ & 15 & $12,8 \%$ & \\
\hline
\end{tabular}

Verificou-se que a maioria $(56,6 \%)$ era do sexo feminino; a faixa etária mais presente no serviço foi entre 60 e 69 anos (53,3\%), com média de 70,0 e desvio-padrão de 7,1. Outra variável significativa identificada entre os cadastrados do PSF foi a baixa escolaridade, e ressalta-se que a quase metade $(47,2 \%)$ dos idosos desse serviço era analfabeta. No que se refere ao estado civil, chama a atenção o fato de $41 \%$ dos idosos serem viúvos e $14,2 \%$, solteiros, o que sinaliza para um percentual significativo de idosos $(55,2 \%)$ morando sozinhos.

Quanto ao gênero, identificou-se proporções mais elevadas do sexo masculino que eram alfabetizados, casados e que viviam com esposas $(p<0,05)$. 
Tabela 2 - Distribuição dos Idosos segundo as variáveis relacionadas ao uso da medicação entre os Idosos cadastrados em PSF na cidade de Montes Claros, 2008

\begin{tabular}{|c|c|c|c|c|c|c|c|c|}
\hline \multirow{3}{*}{ Variável } & \multicolumn{4}{|c|}{ Gênero } & \multirow{3}{*}{ p-valor } & \multirow{3}{*}{$O R$} & \multicolumn{2}{|c|}{$\begin{array}{c}\text { Intervalo de } \\
\text { Confiança }\end{array}$} \\
\hline & \multicolumn{2}{|c|}{ Masc ulino } & \multicolumn{2}{|c|}{ Fe minino } & & & Limite & Limite \\
\hline & $\mathbf{n}$ & $\%$ & n & $\%$ & & & Inferior & Superior \\
\hline \multicolumn{9}{|l|}{ Uso de me dicam entos } \\
\hline Não usa & 14 & $15,2 \%$ & 11 & $9,2 \%$ & \multirow{2}{*}{0,176} & \multirow{2}{*}{1,78} & \multirow{2}{*}{0,77} & \multirow{2}{*}{4,13} \\
\hline Usa & 78 & $84,8 \%$ & 109 & $90,8 \%$ & & & & \\
\hline Não usa corre tam ente & 21 & $26,6 \%$ & 32 & $29,4 \%$ & \multirow{2}{*}{0,676} & \multirow{2}{*}{0,87} & \multirow{2}{*}{0,46} & \multirow{2}{*}{1,66} \\
\hline Usa c orre tam ente & 58 & $73,4 \%$ & 77 & $70,6 \%$ & & & & \\
\hline \multicolumn{9}{|c|}{ Recebeu orientação no uso da medicação } \\
\hline $\operatorname{Sim}$ & 58 & $74,4 \%$ & 88 & $81,5 \%$ & \multirow{2}{*}{0,243} & \multirow{2}{*}{0,66} & \multirow{2}{*}{0,33} & \multirow{2}{*}{1,33} \\
\hline Não & 20 & $25,6 \%$ & 20 & $18,5 \%$ & & & & \\
\hline \multicolumn{9}{|c|}{ Apresenta dificuldade no uso da medicação } \\
\hline Sim & 19 & $24,4 \%$ & 27 & $25,0 \%$ & \multirow{3}{*}{0,920} & \multirow{3}{*}{0,966} & \multirow{3}{*}{0,49} & \multirow{3}{*}{1,90} \\
\hline Não & 59 & $75,6 \%$ & 81 & $75,0 \%$ & & & & \\
\hline \multicolumn{5}{|l|}{ Os medicamentos são: } & & & & \\
\hline Uso contínuo & 64 & $82,1 \%$ & 90 & $83,3 \%$ & \multirow{2}{*}{0,524} & \multirow{2}{*}{0,766} & \multirow{2}{*}{0,34} & \multirow{2}{*}{1,74} \\
\hline Uso oc asional & 13 & $16,7 \%$ & 14 & $13,0 \%$ & & & & \\
\hline
\end{tabular}

0 uso de medicamentos foi comum na maioria dos idosos ( $>90 \%)$. As dificuldades no uso da medicação foram relatadas por menos de $25 \%$ dos idosos. A maioria dos idosos $(>75 \%)$ referiu ter recebido orientações no serviço de saúde sobre a medicação. Verificou-se associação estatisticamente significante para "ausência de dificuldades no uso do medicamento" e "orientações do serviço de saúde (PSF)" $(\mathrm{P}<0,001)$.

Quando aplicados os testes estatísticos para verificar a correlação do uso de medicação com as variáveis sexo, idade, estado civil e com quem mora, observou-se a associação estatisticamente significante para estado civil $(P<0,001)$, escolaridade $(P<0,003)$ e com quem mora $(P<0,001)$, reforçando que essas variáveis são importantes no quesito uso de medicamentos entre idosos.

$\mathrm{Na}$ Tabela 2 observa-se que não há diferenças entre os sexos quanto ao uso de medicamentos, uso correto, orientações recebidas, dificuldades referidas no uso ou tipo de medicamentos. No entanto ressaltam-se os seguintes resultados relacionados ao sexo:

há um maior percentual de casados entre os pacientes do sexo masculino, em comparação às pacientes do sexo feminino; há um maior percentual dos demais estados civis entre as pacientes do sexo feminino;

- há um maior percentual de alfabetizados entre os pacientes do sexo masculino do que entre os do sexo feminino;

- há um maior percentual de pacientes que moram com as esposas, entre os homens, e um maior percentual de pacientes que moram com os filhos entre as pacientes do sexo feminino.

Verificou-se associação estatisticamente significativa para as variáveis "dificuldade no uso" e "uso contínuo" ( $P<$ $0,001)$. As morbidades mais referidas foram: hipertensão $(79,7 \%)$, problemas do coração (12,3\%), dores (15\%) e problemas relacionados ao Sistema Nervoso Central (10,7\%). As variáveis "medicamentos utilizados" e "morbidades associadas" $(\mathrm{P}<0,001)$ mostraram-se associadas estatisticamente.

Quanto aos medicamentos de uso comum entre os idosos entrevistados, os mais utilizados foram: Captropil (39\%), Hidroclorotiazida $(29,4 \%)$, Nifedipina $(16,6 \%)$, Propanolol $(15,5)$ e AAS $(9,1 \%)$; já as morbidades mais apresentadas: hipertensão (79,7\%), problemas do coração $(12,3 \%)$, dores (15\%) e problemas relacionados ao sistema nervoso central $(10,7 \%)$.

\section{DISCUSSÃO}

No presente estudo foi possível descrever o uso de medicação segundo gênero na população idosa cadastrada em um Programa Saúde da Família. Os resultados demonstraram que as idosas recebem mais orientações do serviço de saúde, são mais medicalizadas e usam mais incorretamente as medicações em comparação com os idosos do sexo masculino.

Nesse estudo, assim como identificado por outros autores $^{12}$, identificou-se maior número de mulheres entre os idosos. As mulheres procuram mais os serviços de saúde, 
alimentam-se melhor, fumam menos e consomem menos bebida alcoólica ${ }^{11}$. Outros estudos ${ }^{9-10}$ afirmam que elas estão mais atentas aos sintomas físicos e dispensam mais atenção aos problemas de saúde. Dados do Instituto Brasileiro de Geografia e Estatística (IBGE) ${ }^{12}$ mostram que a mulher vive, em média, 7 anos a mais que os homens, ${ }^{9}$ dado também identificado em trabalho já realizado ${ }^{5}$.

Os idosos com idade entre 70 e 79 anos (35,4\%) indicaram o aumento da longevidade e, ainda, que essa presença nos serviços de saúde é associada ao acesso advindo da implementação do Programa de Saúde da Família, que localizou os idosos em suas residências ${ }^{5,12}$.

Quanto aos aspectos educacionais dos idosos cadastrados nesse PSF, os dados corroboram as estatísticas nacionais sobre educação no País ${ }^{5}$. que revelaram que um percentual significativo não teve acesso aos serviços educacionais. Considerando-se que as variáveis educação e saúde devem ser articuladas, o analfabetismo pode comprometer a qualidade da saúde desses idosos pela falta de conhecimento, compreensão e cuidados na promoção de sua saúde ${ }^{13}$. Ressalta-se que a informação referente ao alto índice de analfabetos entre os idosos cadastrados nesse PSF mostra que existe espaço importante para implementação de políticas de educação para adultos nesse território ${ }^{14}$.

Quanto às diferenças entre os sexos, verificou-se que apenas $38,3 \%$ das mulheres eram alfabetizadas em comparação com $58,7 \%$ dos homens. Esse estudo demonstra que as mulheres encontram-se em situações menos privilegiadas do que os homens em relação à escolaridade. Esse fato pode ser explicado pelas seguintes questões: as mulheres possuem uma sobrecarga de funções visto que tem um papel importante no núcleo familiar, exerce funções domésticas e possuem atividades externas ao domicílio.

Em estudo sobre "Aspectos Epidemiológicos de Idosos Assistidos pelo Programa de Saúde da Família (PSF)", encontraram baixa escolaridade da maioria dos participantes do estudo, assim $43,1 \%$ eram analfabetos ${ }^{13}$. Em um estudo sobre avaliação global de idosos, verificaram que foram predominantes as condições de não saber ler nem escrever e de relativamente pouco tempo de escolarização entre os idosos $(56,7 \%)^{14}$. Em estudo sobre o "Uso de serviços básicos de saúde por idosos portadores de condições crônicas", encontraram na Região Sul a maioria dos idosos com algum grau de escolaridade, e na Região Nordeste a maioria nunca havia frequentado a escola ${ }^{15}$.

No item "com quem moram os idosos", 55,2\% moram sozinhos. Morar com familiares nem sempre é sinônimo de segurança e cuidado adequado para esse grupo. Com frequência, na mídia, ouvem-se relatos de maus tratos com idosos, e, muitas vezes, esses maus tratos envolvem pessoas da própria família. Estudos indicam que nem sempre os familiares no Brasil cuidam bem dos seus idosos, e esta pode ser uma sinalização de preocupação. A esse respeito, ressalta-se que, infelizmente, ainda não temos consolidada uma política pública eficiente para o idoso, como as comuns nos países desenvolvidos. Quanto ao gênero, não houve diferenças relevantes.

Há um maior percentual de casados entre os pacientes do sexo masculino, em comparação às pacientes do sexo feminino; há um maior percentual de pacientes que moram com as esposas entre os homens e um maior percentual de pacientes que moram com os filhos entre as pacientes do sexo feminino.

A este respeito, vale ressaltar que, no cotidiano, é mais comum observar mulheres em idade mais avançada sozinhas. Isso pode estar associado à questão cultural e ao fato de homens, em geral, serem mais dependentes de ajuda, sobretudo nas questões do cuidado. Além do mais, ressalta-se que, historicamente, as mulheres exercem mais a função de "cuidadoras", o que faz com que, na velhice, elas não tenham tanta dificuldades de cuidarem de si próprias; o mesmo não acontece com os homens, que procuram sempre uma companheira para fazer este papel. Por isso, na pesquisa, observou-se que há mais mulheres viúvas, separadas e morando sozinhas ou com filhos. Quando homens precisam de companhia, é mais comum procurarem "esposas", enquanto as mulheres, quando precisam de companhia, mais comumente optam pela companhia dos filhos.

Há um maior percentual de alfabetizados entre os pacientes do sexo masculino do que entre as do sexo feminino. No passado, as mulheres tinham menos acesso aos serviços educacionais, sobretudo em regiões vulneráveis como é o caso da Região Norte de Minas Gerais, o que leva a pesquisa a identificar um maior número de mulheres analfabetas em detrimento de homens. Isso pode também estar relacionado ao fato de as mulheres, quando mais jovens, dedicarem grande parte de seu tempo ao lar, ao cuidado com filhos e maridos, 0 que as afastam do ambiente escolar, fazendo com que, na velhice, esta situação se revele como exclusão educacional entre elas.

A renda é um aspecto importante das condições de saúde e uma variável importante para se medir o grau de desenvolvimento humano ${ }^{13}$. Os resultados deste estudo apontam que esses idosos apresentam renda insuficiente para arcar com as demandas pertinentes à idade; sobretudo, as demandas de saúde. A respeito dessa questão, ressalta-se que são bastante comuns os relatos dos idosos de que sua aposentadoria ou renda restringe-se à compra de medicamentos, o que é ancorado por estudos que demonstram que os gastos com medicamentos têm um peso importante no orçamento da população idosa brasileira - metade dela recebe mensalmente menos de um salário mínimo e gasta em média $23 \%$ dessa renda na aquisição de medicamentos, e 0 abandono do tratamento medicamentoso em decorrência de seu custo é comum $^{16}$.

Considerando-se o ciclo vital, o idoso está na faixa em que se precisa alimentar adequadamente, realizar atividades 
físicas inerentes à idade, frequentar, com certa regularidade, os serviços de saúde, e rendas inferiores a três salários mínimos podem comprometer tais demandas $2,4,11,14$.

No que se refere às principais dificuldades apresentadas pelos idosos no uso de medicamentos, a maioria $(75,6 \%)$ informou que não apresenta dificuldade. Quando se procurou conhecer as razões para a ausência de dificuldades no uso da medicação entre esses idosos, verificou-se que um fator que contribui para essa situação é a orientação dos serviços de atenção primária à saúde. Em relação a essa informação, ressalta-se que foi encontrada associação estatisticamente significante $(P<0,001)$ para "ausência de dificuldades no uso do medicamento" e "orientações do serviço de saúde (PSF)".

A maioria dos idosos que informou "não ter dificuldades no uso do medicamento" também informou "ter tido orientação quanto ao uso por parte de algum profissional da atenção primária à saúde". Esse dado é relevante porque reforça a importância da orientação, sobretudo para a população idosa que, como já foi mencionado, encontra-se em um estágio da vida que requer maior atenção e cuidados. Em especial, quando há dificuldades na identificação de medicamentos, pode promover o uso inadequado, além de causar danos que comprometam ainda mais sua saúde.

0 "esquecimento" é apontado por outros autores como a principal dificuldade no uso correto da medicação, pois o idoso, normalmente, apresenta déficit de memória. A esse respeito, existem argumentos de que "as dificuldades em se obter adesão ao tratamento quase sempre é agravada pelo 'déficits' de memória e visual que dificultam distinguir e manipular diversos medicamentos ao mesmo tempo". Estudos apontam que o uso inadequado de medicamentos entre idosos, com frequência, provoca complicações graves ${ }^{17: 58}$. Embora se reconheça a importância de se elucidar e entender em profundidade as principais dificuldades apresentadas pelos idosos em relação ao uso de medicamentos, ressalta-se que foi objetivo do presente estudo apenas identificá-las, podendo um estudo futuro tratar especificamente desta questão.

Por meio de testes estatísticos, foi possível verificar uma associação estatisticamente significante com o estado civil e a ausência de dificuldade no uso do medicamento $(\mathrm{P}<$ $0,001)$. A esse respeito, ressalta-se que a maioria dos idosos sem dificuldade é casada ou vive com companheiro. Assim, infere-se que a presença do cônjuge pode ser um aspecto importante para a administração correta do medicamento, pois, se o idoso apresentar dificuldade, o companheiro poderá auxiliálo. Este dado foi apresentado nos resultados: preponderância de sexo masculino com companheiro (Tabela 1 e Discussão).

A pesquisa e os testes estatísticos evidenciaram, também, associação estatisticamente significativa para "dificuldade no uso" e "uso contínuo" ( $P<0,001)$; ou seja, "aqueles que informaram ter dificuldades no uso" em sua maioria "são os que fazem uso contínuo de medicamento". 0 uso diário pode trazer a banalização e o esquecimento se não
Silva CSO, Pereira MI, Yoshitome AY, Neto JFR, Barbosa DA

houver uma estratégia estabelecida para não confundir com o uso do dia anterior ${ }^{18}$.

Embora não tenha sido explorado na pesquisa (e nem apresentado no corpo do artigo), considerou-se importante um achado: a pesquisa evidenciou uma associação estatisticamente significante entre medicamentos utilizados e morbidades associadas $(P<0,001)$; ou seja, existe uma estreita relação entre os medicamentos mais utilizados e as morbidades apresentadas pelos idosos. Este pode ser um enfoque a ser explorado em uma outra pesquisa.

Merece destaque o fato de os idosos entrevistados utilizarem, majoritariamente, medicamentos disponíveis no sistema público de saúde para suas morbidades. Isso demonstra 0 acesso aos medicamentos por meio dos programas de farmácias básicas e populares, que têm sido implementados no País. 0 fato de os idosos utilizarem medicamentos do serviço público ameniza ou neutraliza um pouco a utilização de sua renda com a compra de medicamentos. As morbidades apresentadas coincidem com o perfil de morbidade do País, que apresenta as doenças crônicas no rol principal do adoecimento da população brasileira ${ }^{15,18}$.

Os resultados devem ter sua interpretação considerando algumas limitações. As limitações do estudo referem-se ao espaço geográfico limitado (abrangência de 01 PSF) e às dificuldades de se entrevistar o idoso.

\section{CONSIDERAÇÕES FINAIS}

Por meio deste estudo foi possível chegar a algumas considerações importantes tanto em relação às características do idoso, quanto em relação ao comportamento frente ao uso de medicamentos por parte desta população no município de Montes Claros, Minas Gerais. Num universo de 228 idosos cadastrados em um PSF deste município, verificou-se maioria (56,6\%) do sexo feminino; maior frequência registrada no serviço para a faixa etária entre 60 e 69 anos (53,3\%), baixa escolaridade: quase metade $(47,2 \%)$ dos idosos desse serviço é analfabeta. 0 uso de medicamentos foi comum na maioria dos idosos (>90\%). Já as dificuldades no uso da medicação foram relatadas apenas por menos de $25 \%$ dos idosos, sendo que estas dificuldades foram minimizadas por meio de orientação quanto ao uso de medicamento por parte de algum profissional da atenção primária à saúde", uma vez que a maioria dos idosos (>75\%) referiu não ter dificuldade exatamente por ter recebido tal orientação, evidenciando associação estatisticamente significante para "ausência de dificuldades no uso do medicamento" e "orientações do serviço de saúde (PSF)". Assim sendo, considera-se que este estudo poderá subsidiar políticas públicas de atenção ao idoso, sobretudo enfatizando a importância da educação em saúde para este estrato populacional. 


\section{REFERÊNCIAS}

1.Ministério da Saúde (BR). Estatuto do Idoso. $2^{\text {a }}$ ed. Brasília (DF); 2009. 70p.

2.Organização Pan-Americana da Saúde- OMS. Rede Interagencial de Informações para Saúde. Demografia e saúde: contribuição para análise de situação e tendências. Brasília (DF); 2009.

3.Malta DC, Duarte EC. Causas de mor tes evitáveis por ações efetivas dos serviços de saúde: uma revisão da literatura. Cienc Saude Colet [periódico na Internet], 2007 jun [citado 201006 mar 06];12(3): 765-76.

Disponível em: http://www.scielo.br/pdf/csc/v12n3/27.pdf.

4.Camarano AA. Envelhecimento da população brasileira: uma contribuição demográfica. Brasília (DF): IPEA; 2002.

5.Giacomin KC, Uchoa E, Firmo JOA, Lima-Costa MF. Projeto Bambuí: um estudo de base populacional da prevalência e dos fatores associados à necessidade de cuidador entre idosos. Cad Saude Publica 2005 fev; 21(1): 80-91.

6.Veras R. Fórum envelhecimento populacional e as informações de saúde do PNAD: demandas e desafios contemporâneos. Introdução. Cad. Saude Publica; 2007 out; 23(10): 2463-66.

7.PazAA, Santos BRL, Eidt OR. Vulnerabilidade e envelhecimento no contexto da saúde. Acta Paul Enferm. 2006 jul/set; 19(3): 338-42.

8.Duarte YAO, Diogo MJD. Atendimento domiciliar: um enfoque gerontológico. São Paulo (SP): Atheneu; 2000. 349-64.

9. Teixeira JJV, Lefèvre F. A prescrição medicamentosa sob a ótica do paciente idoso. Rev Saude Publica. [periódico na internet]. 2001 abr [citado 2009 maio 03]; 35(2): 207-213. Disponível em: http://www.scielo.br/ $\mathrm{pdf} / \mathrm{rsp} / \mathrm{v} 35 \mathrm{n} 2 / 4407 . \mathrm{pdf}$.

10.Rozenfeld S. Prevalência, fatores associados e mau uso de medicamentos entre os idosos: uma revisão. Rev Saude Publica 2003; 19(3): 717-24.

11.Ministério da Saúde (BR).Vigitel Brasil 2006: vigilância de fatores de risco e proteção para doenças crônicas por inquérito telefônico. Brasilia (DF); 2007. 92p.

12.Instituto Brasileiro de Geografia e Estatistica- IBGE. Perfil dos idosos responsáveis pelos domicílios. Rio de Janeiro (RJ); 2002 [citado 2010 mar 06]. Disponível em: http://www.ibge.gov.br/home/presidencia/noticias/ 25072002pidoso.shtm.

13.Lopes FAM, Oliveira FA. Aspectos epidemiológicos de idosos assistidos pelo Programa de Saúde da Família- PSF. Textos Didáticos do Curso de Especialização em Saúde da Família e Disciplina de Patologia Geral da Faculdade de Medicina do Triângulo Mineiro- FMTM. Uberaba (MG): FMTM; 2004.

14.Marin MJS, Cecílio LCO, Rodrigues LCR, Ricci FA, Druzian SD. Diagnósticos de enfermagem de idosas carentes de um Programa de Saúde da Família- 